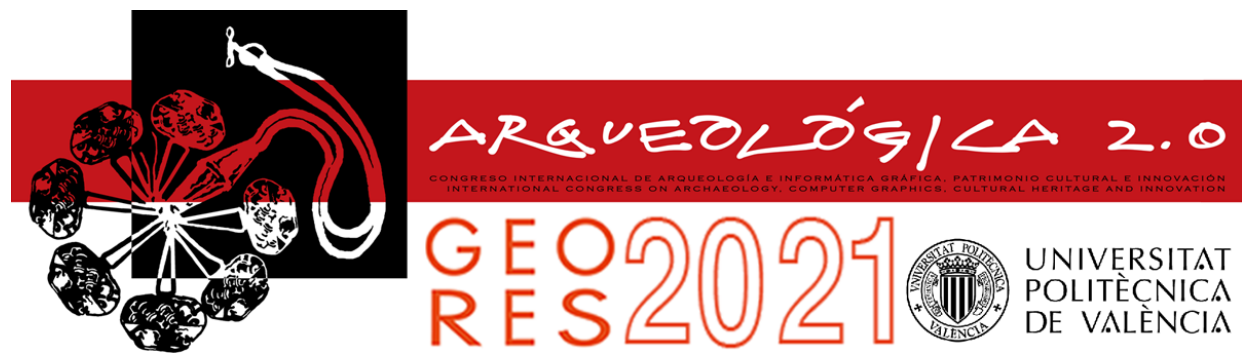

\title{
PARAMETRIC GENERATION IN HBIM WORKFLOWS FOR SLAM-BASED DATA: DISCUSSING EXPECTATIONS ON SUITABILITY AND ACCURACY
}

\author{
Giulia Sammartano ${ }^{a, b,}{ }^{,}$, Mattia Previtalic, Fabrizio Banfic \\ a Department of Architecture and Design (DAD), Politecnico di Torino, Viale Mattioli 39, 10125 Torino, Italy. giulia.sammartano@polito.it \\ ${ }^{b}$ Future Urban Legacy Lab (FULL), Politecnico di Torino, Toolbox coworking, Via Agostino da Montefeltro 2, 10125 Torino, Italy. \\ c LAB Gicarus, Department of Architecture, Built Environment and Construction Engineering (ABC), Politecnico di Milano, Via G. Ponzio \\ 31, 20133 Milano, Italy. fabrizio.banfi@polimi.it; mattia.previtali@polimi.it
}

\begin{abstract}
:
In the wide framework of Scan-to-BIM 3D modelling procedures, the complexity of the architectural heritage and its components is evidencing a gap of best practices and specification in the HBIM-modelling and thus it is requiring important considerations about the modelling strategies and protocols between the requested level of detail (LOD), the expected accuracy and above all the actual user-oriented requirements. Several works are largely focusing on developing modelling workflows for traditional static LiDAR scanning sensors. The amount of starting data is as much an asset when a bottleneck. In fact, the chance to benefit from on-site faster data acquisition is needful at times, and data collection procedures are directing toward rapid mapping 3D approaches, evolving from traditional static scanning toward MMS (Mobile Mapping Systems) based on SLAM technology (Simultaneous Localization and Mapping) implemented in portable devices. The potential derived from these solutions can contribute to increasing massive cost-effective documentation, and also in view of BIM-HBIM models generation, and this needs further researches. At the same time, the descriptive capabilities of this class of portable scanners do not reach the precision of static solutions. Many considerations towards the analysis of geometry, grade of generation (GOG) and details can be thus conducted, especially under the point of view of time-cost balance. This paper aims to presents the entire modelling workflow (from raw data acquisition up to parametric modelling) based on a first comparison between TLS (Faro Focus 3D) and hand-held scanner Zeb Revo (by GeoSLAM), focusing on the Bramante's Canonica Court in the Basilica of Sant'Ambrogio. First, the two raw data are compared, considering geometric features (data density, precision, possibility to detect edges, details and accurate curvature). Then, some wellestablished modelling procedures developed for TLS data, as triangulation mesh and NURBS generation, are applied to MMS point cloud to assess their suitability, identifying limits and potential. Different elements belonging to the architectural structure hierarchy are considered in a multi-scale perspective: the vaulted system of the porch, the columns and the arches of the porch with their different architectural elements.
\end{abstract}

Keywords: SLAM, MMS, grade of accuracy, grade of generation, HBIM, TLS, model accuracy, model scales

\section{Introduction}

In the last decade, great attention was paid to the topic of defining clear and reliable workflows for incorporating 3D laser scanning and photogrammetric point cloud information within a BIM environment (Oreni et al., 2014; Rocha, Mateus, Fernández, \& Ferreira, 2020; Yang, Lu, Murtiyoso, Koehl, \& Grussenmeyer, 2019). Indeed, in recent times two different trends have been observed: on the one side the noticeable emergence of technology supporting the integration of rich data within architectural models in the framework of building information modelling (BIM) and on the other side the rapid expansion on surveying technologies (e.g., 3D laser scanning and photogrammetry) that allowed fast and reliable digitization of large and complex architectures. The adoption of BIM paradigm proved to be revolutionary in many applications like design (Wong \& Fan, 2013), construction site management (Kumar \& Cheng, 2015) and maintenance (Motawa \& Almarshad, 2013) among others. The BIM concept shows great potentialities also in the case of Historical buildings (HBIM) where well-established and shared concepts within building and materials conservation can be associated with specific building objects that may be characterized by complex and irregular geometries. Here the potential of scanning technologies (active or passive) to record the existing built environment is clear: complex shapes, irregular geometries and geometrical anomalies that characterize historical building can be easily recorder with level of accuracy which was not feasible even a couple of decades years ago. Even if the cross-connection between BIM and $3 \mathrm{D}$ scanning is evident, the development of a

*Corresponding Author: Giulia Sammartano, giulia.sammartano@polito.it 
workflow for effective and efficient integration of laser scan data within a BIM environment has required an indepth study and several types of research are focusing on this issue (Dore \& Murphy, 2013; Hichri, Stefani, De Luca, Veron, \& Hamon, 2013; Oreni et al., 2014; Rocha, Mateus, Fernández, \& Ferreira, 2020; Yang, Lu, Murtiyoso, Koehl, \& Grussenmeyer, 2019). Indeed, even if it is possible to import point clouds within BIM software, in order to make the best use of the highly accurate and often massive data, a certain amount of postprocessing (filtering, smoothing, subsampling, etc.) is strictly required. The amount of acquired data is as much an asset when a bottleneck. In addition, modelling strategies should be adopted and often adapted to transform a point cloud into parametric objects. The different Level of Geometry (LOG) that are connected with the different Level of Development (LOD) can be obtained by using different strategies. In particular, the possibility offered by different Grades of Generation (GOG) and the possibility to use NURBS (GOG 9-10) proved to be successful to accurately represent complex geometries in BIM applications (Banfi, 2017). On the other hand, solid parametrization modelling and GOG 1-8 can be successfully used for LOG and LOD characterized by a lower level of details and wherever high accuracy is not required. Nowadays consolidated workflows have been developed for Scan-to-BIM and are efficiently used in several projects. The issue on the treatment of the starting massive data remains open.

\subsection{Motivation}

Reality-based modelling is highly benefitting from scanning data in the so-called as-built modelling. Even if point clouds can be directly used for the production of BIM objects (e.g., GOG 9-10) the amount of data really used for the modelling is much lower with respect to the one acquired (Fig. 1). Point clouds are highly subsampled and only a small fraction of the original data is used: often less than $1 / 10$. Due to this aspect, a high percentage of the points is not directly used in surface generation making them unused in the case of parametric modelling. This leads to a contradiction: on the one side highly density point clouds are collected in the surveying stage (in order also to guarantee the required precision in scan registration) on the other hand the majority of those points is not used in the surface generation and modelling workflow. As a consequence of that, the survey with traditional static Terrestrial Laser Scanning (TLS) may be considered by then as not the optimal data source and survey method for all applications. Indeed, even if a TLS survey is much faster than traditional survey techniques and allows to capture structures that are well outside the physical reach of the surveyors, a TLS campaign may be quite a time expensive, some hours to few days according to the extent of the area, especially in the case of complex structures to avoid occlusions and high completeness with homogeneity of points distribution.

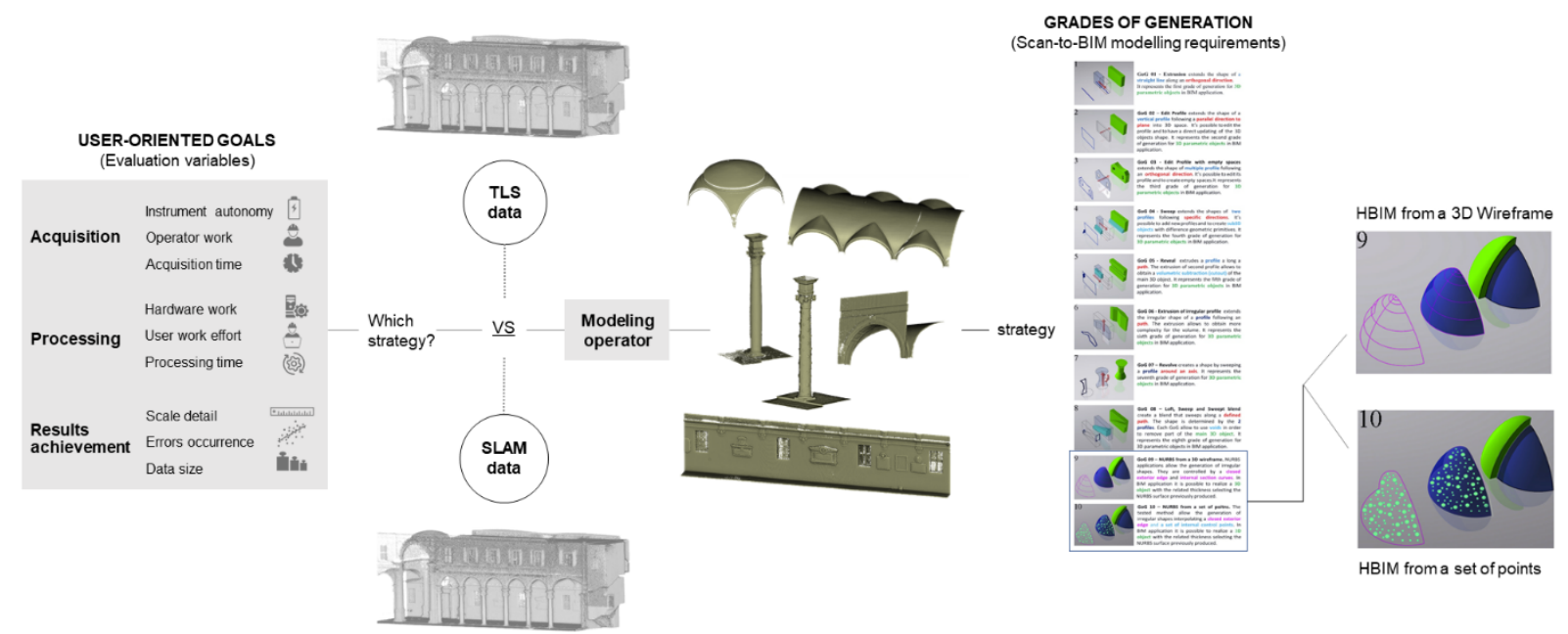

Figure 1: The diagram indicates the research demand, through the investigation of suitable strategies. The established HBIM parametric modelling is applied to SLAM-based data according to a series of user-oriented variables. The investigated generation approaches are the GOG9 and GOG10.

In the last years, the availability of Mobile Mapping Systems (MMS) proved to be an interesting alternative to TLS for a large set of applications, especially if portable as handheld or backpack tools, like: rapid mapping after earthquakes and extreme events (Sammartano, 2018), the survey of industrial sites, quarries and mines (Dewez, Yart, Thuon, Pannet, \& Plat, 2017), narrow spaces (Zlot \& Bosse, 2014) and heritage and archaeological sites (di Filippo et al., 2018; Sammartano \& Spanò, 2018; Spanò, 2019; Zlot et al., 2014). The main advantage of those instruments is the "scan-while-walking", making the survey of large areas more efficient with respect to the traditional TLS survey, in terms of time-saving. In a general way, a handheld Indoor Mobile Mapping system is composed by a $2 \mathrm{D}$ infrared laser scanner profilometer generally coupled with an IMU sensor. The laser profilometer continuously provides the range measurement, while the IMU sensor, generally triaxial gyros and accelerometers, which is firmly mounted close to the laser, provides measurements of angular velocities and accelerations. The IMU sensor and the scanned 2D profile data are used to estimate the sensor position and progressively the trajectory. The $2 \mathrm{D}$ laser profiles acquired by the profilometer are continuously aligned by using 3D SLAM (simultaneous localization and mapping) approach (Riisgaard \& Blas, 2005), estimating the six degrees of freedom of the sensor motion, and generates the $3 \mathrm{D}$ point cloud of the scene. The more the scanned environment is rich in detail with no recurring elements, 
the more the SLAM algorithms benefits from the geometric features extracted during the operating.

The main drawbacks of MMS compared with TLS are: i) lower point density and ii) lower precision in point measurement. Indeed, LiDAR mounted on MMS are characterized by single-point measurements precision of some centimetres $(1-3 \mathrm{~cm})$. An extensive review of MMS and comparison of MMS and TLS data is out of the scope of the presented work, for more details the reader is addressed to (Nocerino, Menna, Remondino, Toschi, \& Rodríguez-Gonzálvez, 2017; Sammartano \& Spanò, 2018; Tucci, Visintini, Bonora, \& Parisi, 2018).

This paper aims to investigate, from an end-user perspective, the possibility of using MMS data for modelling specific architectural elements that can be interoperable with BIM objects and not customary in the current application. The main purpose of the work is to verify the possibility to use a more time-cost efficient workflow related to end-user's requirements in terms of requested accuracies, level of details and expected scale. In particular, the main question we would like to address can be summarized as follows: "Which are the main differences in the TLS/MMS data acquisition and processing workflows? Which are the differences in terms of data density, precision, possibility to detect details and curvature between TLS and MMS? Which accuracy and level of details can be obtained in the parametric modelling? The modelling strategies (GOG) used for TLS can be adopted also for MMS?"

\section{Multi-scale architectural elements modelling strategies}

The reality-based modelling from point clouds, as introduced, is essentially based on a continuous geometry reconstruction, with or without semantic information association, from processes of understanding, simplification, approximation and interpolation of the starting 3D data. The modelling pipeline cannot be completely separated from human interaction and interpretative intervention. Uniqueness, richness, complexity and anomalies of architectural elements are the crucial issues to be featured in their geometric, radiometric and semantic dimensions' definition for a pre-determined scope for which the informative model is designed and expected. But they don't always have all the same weight: the end-useroriented requirements are the constraints on which to base the modelling organization ex-ante (Banfi, 2017). For this reason, the pure modelling based on NURBS, solid volumes and shapes from scratch generation, which proves not to be very flexible and adaptive to the specificities of existing heritage shapes, is usually set aside to make way for point-cloud based modelling, aroused from the as-built modelling. The polygonal mesh model Figure $2 a$ is a first-step solution of transformation from unorganized points set via interpolation of a continuous surface made of triangles or quadrangles (Remondino, 2003). In this case, the solution leads to a continuous surface and not solid object, often with radiometric content deriving from textures, even with very impressive photorealistic visualization effects, generated by photogrammetric approaches but not semantically organized.
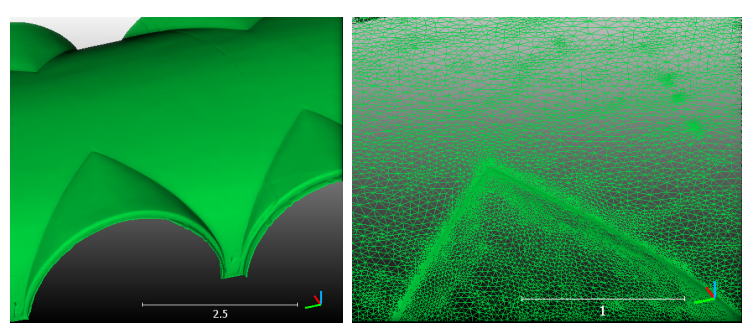

(a)
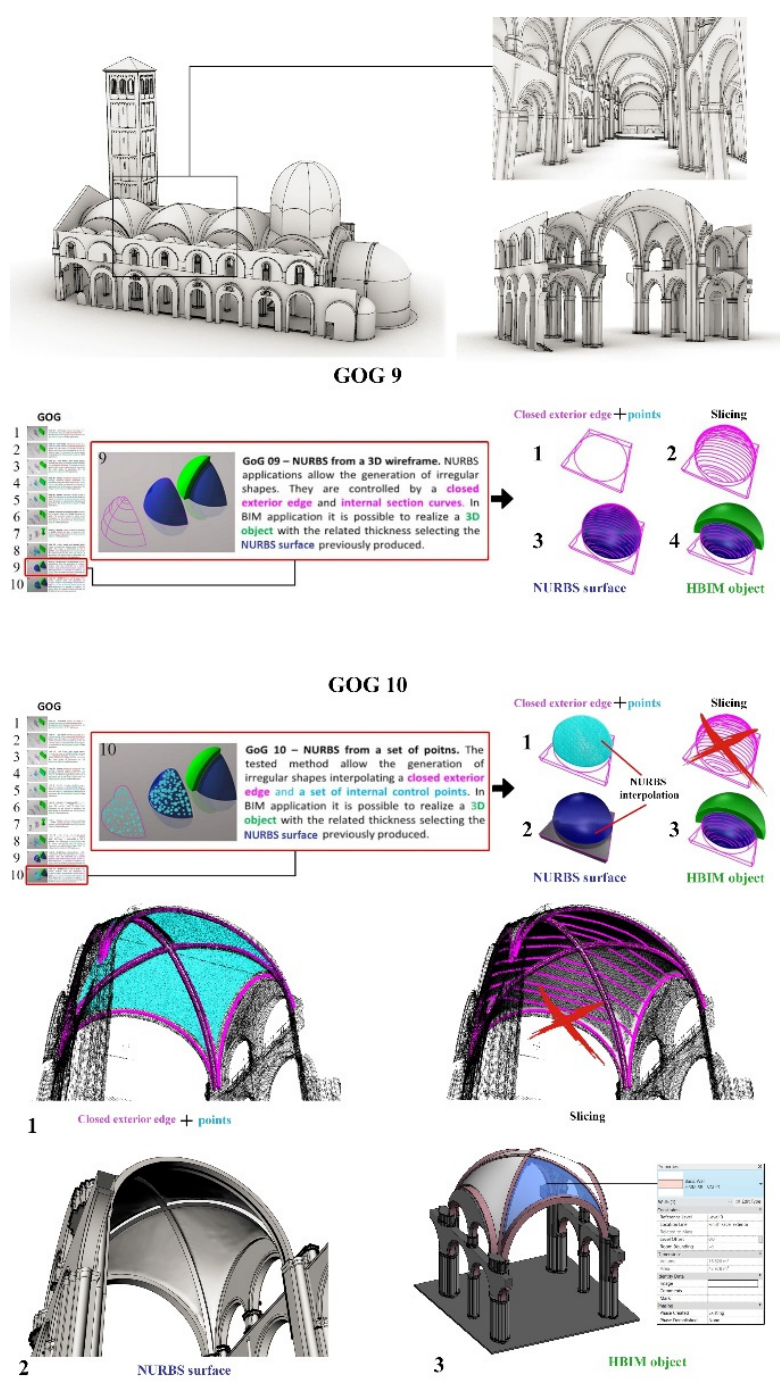

(b)

Figure 2: The 3-types modelling step, based on point cloud data operated in the vaulted system of the porch in the case study, the Bramante Canonica court: (a) the triangulated mesh; (b) a GOG9 based on extracted primitives and edges and a GOG10 based on external edge and interpolation of a points sample.

Contrariwise, the so-called scan-to-BIM approach is characterized, as well known, by the possibility to involve the parametrization procedures rules in the generation of $3 \mathrm{D}$ objects and not only interpolated surfaces. The modelling is therefore constrained based on generative geometries and 3D transformation or combination of them, that are more or less complex and articulated in their hierarchy or Grade of Generation, as developed in (Banfi, 2019) and represented in Figure 2b. GOG are 
dependent on many variables but, first of all, the selection of a starting 3D data is one of the crucial factors affecting the result. It could therefore be said that there is an exante connection between GOG of models and LOD of points models. Afterwards, the needs of explicit geometry generation require different parametric modelling approaches, less or deeply connected with the point cloud detail Figure $2 \mathrm{~b}$, or with specific 3D profiles primitives and edges extracted from it Figure $2 \mathrm{~b}$. As a consequence of recent research developments in the framework of parametric modelling, many operative solutions have been implemented to lead advanced modelling tools in order to extract, extrude, revolve, swipe etc... It can therefore be summarized that the more complex the shape and the LOD is required for the informative model for user-oriented purposes, the more the GOG must be chosen and applied to solve the geometric definition of architectural elements at the proper scale. For this reason, traditionally LiDAR point clouds have always been connected to as-built modelling, for their ability to provide support in the modelling stage with different LODs and GOGs. In this particular context, the degrees of generation and the 3D survey tools are also decisive for exponentially reducing the time and costs related to the creation of as-found models. So Therefore in the presented research, architectural and structural elements have been selected that are able to outline a scan-to-BIM workflow, allowing the authors to compare the application of generation grades 9 and 10 beyond the point clouds (TLS and MMS), the last identified and developed to support the generation of complex elements from point clouds.

The main consistent difference is that the GOG9 (HBIM object from wireframe model) is based on the extraction of profiles and geometric primitives from a point cloud while the GOG 10 automatically transforms a selection of points corresponding to an architectural element into a parametric object Figure 2. However, to achieve an appropriate generation with both of them, it is necessary to identify the 3D edges of the element to be created. For this reason, the identification of the wireframe models corresponding to vaults, walls, columns, arches are determined if the process is based on reliable data. For the GOGs and the scan-to-bim process in general, point clouds are considered primary data sources. Consequently, the more precise a 3D survey is, the more precise the creation of as found models will be. GOGs depend solely on two factors: (i) the accuracy of point clouds and (ii) the user's ability to correctly interpret the construction logic of the surveyed building and the 3D survey data, dividing them into architectural and structural elements (semantic decomposition). Furthermore, at this level, the economic impact linked to the reduction of the number of hours dedicated to every single architectural element is difficult to identify. Many factors within the process need to be considered. On the other hand, in (Banfi, 2020) the generative times associated with GOG 9 and GOG10 have been identified. It was found that the morphology of the element, its semantic breakdown, the information to be associated are the main factors that influence the realization. Consequently, as mentioned in various studies, national regulations and various LODs should consider studies and methods capable of properly explaining the holistic value of HBIM models and the scan-to-BIM process. Different disciplines such as geomatics, representation and restoration now merge into a single digital project. The value of precision of $3 D$ survey, the scale of representation, GOGs and LOD become interconnected. There is not always accurate data like a point cloud coming from TLS or digital photogrammetry. Sometimes, for different project objectives and for non-architectural scales, it is possible to use the integration of other tools. In particular cases, furthermore, within a single HBIM project there may be different LODs, LOIs obtained in turn from 10 different GOGs.

In this context, MMS has been compared to TLS in order to highlight their behaviour in the $3 \mathrm{D}$ mapping of the substantial differences between objects that characterize historical buildings for the most part. Vaults, columns, and arches were detected with both tools and in turn, generated using both GOG9 and GOG10. The final goal indeed is not only to determine what is better tout court but, based on the project objectives and pre-determined LOD, which values derived from TLS or MMS data can be considered the most suitable in terms of precision and accuracy. In other terms, where the use of MMS clouds, due to their competitiveness in terms of time-cost ratio, can support, the achievement of an expected LOD, using 2 different GOGs. Some issues arise based on the employed tools and GOG used to generate the objects. They are reported below in order to clarify the starting point of the study proposed in this article.

If we circumscribe the scale expected from some types of 3D surveys for which the expert user must have an HBIM information model available, can a LOD be satisfied starting from a low precision and high-efficiency point cloud? With what accuracy? The research toward the positive answer must necessarily pass through the control of the GOA of the generated models that can validate the use of such source data.

\section{The case study}

The historical and architectural framework of the $\mathrm{S}$. Ambrogio Basilica particularly stratified site in which the research is conducted leads us to operate on the Bramante's enlargement intervention on the Canonica arcades (portico) of the S. Ambrogio Basilica in Milano dated back to $15^{\text {th }}$ century (Gatti Perer, 1995; Lusuardi Siena, 1997; Marucci, R. A., Negri, M., Rastelli, A. Romaniello, 2004) Figure 3a. The whole remarkable architectural work of Bramante today arrives us after the war damages and the restoration by architect Reggiori in the $20^{\text {th }}$ century. However, the harmonious and cadenced proportions of the arcades of the portico, the classical entasis of columns and finely worked capitals, separated from the arches by pulvini, the columns with gnarled protuberances (laboratas ad tronchonos) still remain (LombardiaBeniCulturali, 2016). They required a tailored approach to their documentation in past years as well as today. Recent researches have dealt extensively with scan-to-bim modelling for the creation of the HBIM information model for various purposes Figure $3 \mathrm{~b}$ (Banfi, Previtali, Stanga, \& Brumana, 2019; Banfi, Brumana, \& Stanga, 2019b; Stanga et al., 2017).

\subsection{Data acquisition \& processing}

The data acquisition belong to different phases. Particularly for the TLS dataset (2014-2015), a great effort has been spent during the last years for the generation of a complete and enriched 3D HBIM model of the whole 
complex of St. Ambrogio, its historical stratification, materials and construction techniques. In 2019, in the occasion of the GEORES Conference and the Young Researcher Workshop event, experimentation of a SLAM-based mapping has been proposed and performed. For the experiment proposed here, the south area of the portico was selected adjacent to the basilica and the bell tower, and the Canonica rooms above Figure 3. A series of considerations will be proposed regarding the different nature of the acquisition and processing pipelines, starting from survey planning, post-processing and cloud optimization. The covered area is almost 2000 $\mathrm{m}^{2}$ on the ground floor and the first floor, for an overall outside and inside volume of $2500 \mathrm{~m}^{3}$.

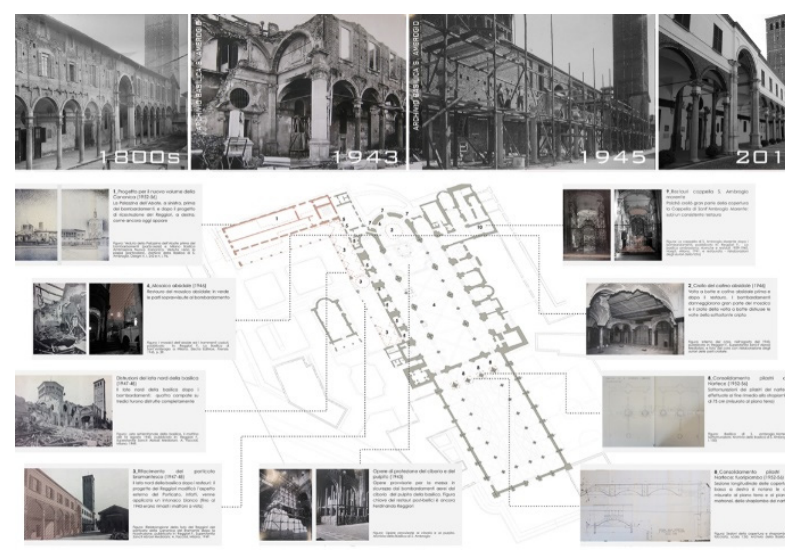

(a)

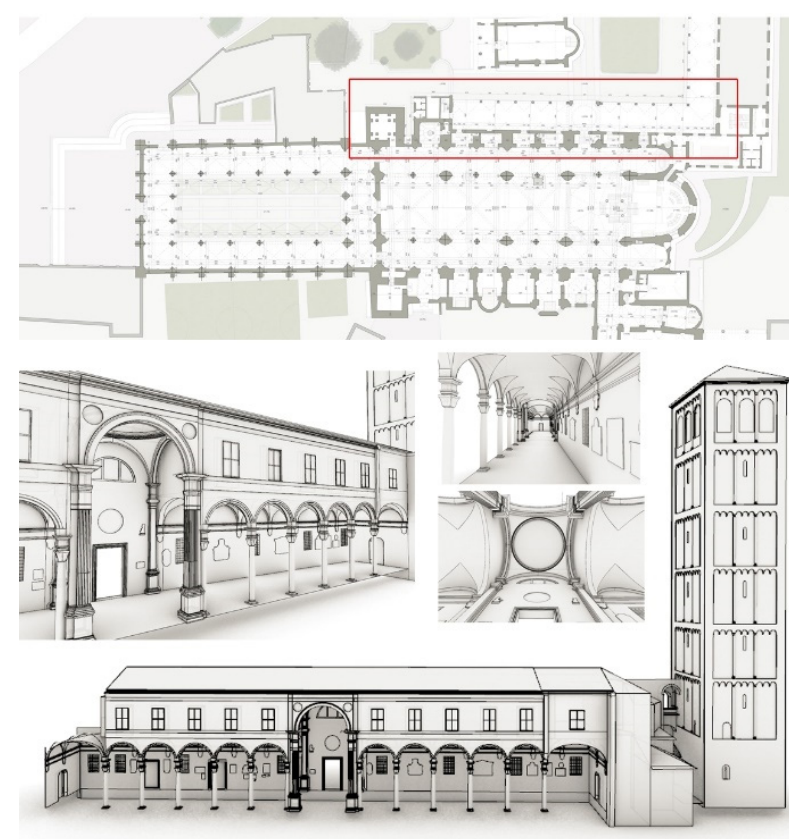

(b)

Figure 3: The case study: the Bramante's Canonica porch in Basilica of Sant'Ambrogio (MI). (a) the history of the building XX cent. across bombing and reconstruction; (b) the architectural drawings generated with the HBIM model recently generated.

\subsubsection{LiDAR points model}

The dataset of $n^{\circ} 20$ scans referred to the Canonica court and upstairs rooms are distributed in the area of the arcade and the first floor designed to cover the visible surfaces, as visible in Figure 4. Supporting the whole 3D metric survey, a geodetic network has been materialized and measured with Total Station Leica TS30. In this network, a set of control points in the form of black \& white target have been measured for the scan's registration and accuracy control. The static laser Faro Focus 3D was employed in different survey phases. The 3D scans were registered with an average precision of $\pm 3.0 \mathrm{~mm}$ by using targets, topographic measures and with additional scanto-scan correspondence (spherical targets) (Banfi, Brumana, \& Stanga, 2019a). The fieldwork required almost 3 hours work for scanning and 40 min for the topographic measures supporting the LiDAR survey (considering already measured topographic network vertices, and only detail survey execution on them), and this is, as usual, a time-consuming procedure. Three expert operators were involved. The average weight of the scans is $800 \mathrm{Mb}$ for coloured scans and $100 \mathrm{Mb}$ for grayscale scans. The time for registration was approximately 4 hours and the derived point model has been cleaned and filtered of about $10 \%$.

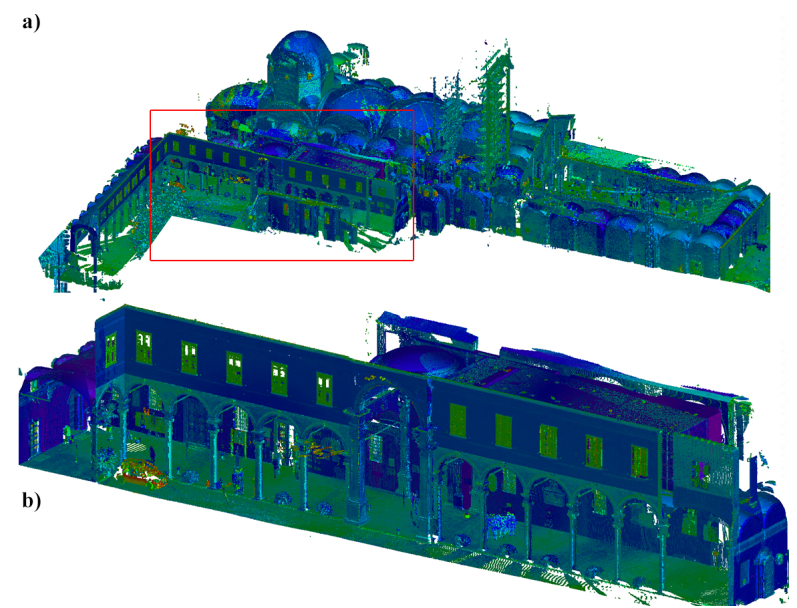

Figure 4: View of the global 3D point cloud from static TLS acquisition on the Basilica (a) and on the Canonica porch (b).

\subsubsection{SLAM-based mapping}

The SLAM acquisition (Fig. 5) was performed using ZEB Revo-RT handheld scanner by GeomSLAM, by $n^{\circ} 1$ expert operator walking almost for $15-20 \mathrm{~min} / \mathrm{scan}$, with the coincidence of starting-ending point. The two clouds, covering the whole area of the arcades and the first-floor rooms were acquired along a trajectory of almost $600 \mathrm{~m}$ roundtrip: $40,7 \mathrm{mln}$ points $+21 \mathrm{mln}$ points, for an entire weight of $970 \mathrm{Mb}$ data. After the acquisition, a first step reprocessing the SLAM algorithm is performed according to a set of parameters and with a non-rigid co-registration of the two scans supporting each other based on 3D features reconstruction (Sammartano \& Spanò, 2018) and finally, the derived point model has been cleaned and filtered of about $20 \%$. The overall amount of time for firststep data re-processing and second step non-rigid coregistration is almost $30+20 \mathrm{~min}$, and in any case less than 1 hour. The third step of manual segmentation and semi-automatic filtering is conducted in almost 2 hours. 


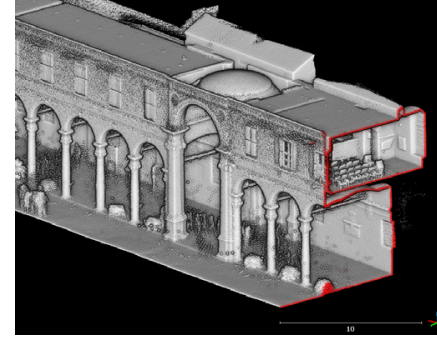

(a)

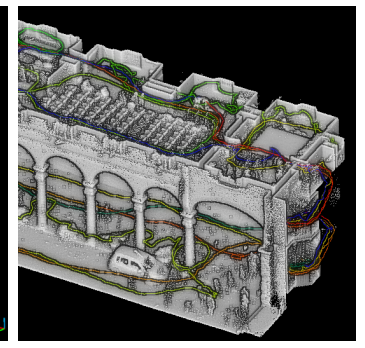

(b)
Figure 5: The SLAM-based point cloud acquired in the two floors levels of the Bramante's Canonica: in a section view (a), with the visualization of the time-marked trajectory (b).

\section{Evaluating SLAM-based points clouds for parametric modelling strategies}

\subsection{Point cloud analysis}

After the first step comparison introduced, related to acquisition procedures of SLAM-based data parallel to traditional static scanners, a second analytic phase is conducted on the points models' quality for parametric modelling and their descriptive capabilities. This phase begins with the metric analysis of the two initial point cloud data and then some specific geometric features are hierarchically considered according to a requirements sequence. Particularly the SLAM-based point clouds are analysed in order to support GOG modelling strategies, according to modelling choices and requirements conducted by the operator during the modelling: GOG 9 based on characterising polylines/break lines, belonging to the objects surfaces, and GOG 10, based on point cloud interpolation and external edge profiles (Banfi, 2019).

Firstly, a global and a local analysis should be considered in terms of accuracy in the case of SLAM acquisition, due to the 3D space reconstruction strictly connected to the trajectory behaviour, as recent researches demonstrated (Nocerino, Menna, Remondino, Toschi, \& RodríguezGonzálvez, 2017; Sammartano \& Spanò, 2018; Tucci, Visintini, Bonora, \& Parisi, 2018). In fact, if generally, the local performance of 3D surface reconstruction of SLAM scanner is suitable for medium-high detail with good continuity of the surface digitization (despite the lower precision of the static Lidar sensor) it is not uncommon that the global accuracy is often affected by drift errors on long distances.

The global accuracy analysis is conducted with TLS scan as a reference, and with SLAM data aligned with a rigid roto-translation fixed in $n^{\circ} 10$ points located closer to the position of the scans start (room adjacent to the side of the stair). The mean deviation error results (Table 1) of the ten points-based alignments is $2,4 \mathrm{~cm}$, with st.dev = $1.3 \mathrm{~cm}$, in line with the results already obtained for this type of verification. In particular, the main error direction is along the $Y$ axe, that is the longitudinal axe, as expected, underlining the influence of scan trajectory direction in the error propagation.

Instead, the global measurement of the drift error far from the starting point and in levels, indicates a deviation error distribution according to both the longitudinal and cross direction of the arcades proportionally to the distance from starting point. In particular, as shown in Figure 6 a horizontal deviation error of SLAM data from TLS data at the ground floor has been identified from $2 \mathrm{~cm}$ up to $4 \mathrm{~cm}$ in the central part and more than $4 \mathrm{~cm}$ in the farthest part from of the porch. The lower error in the horizontal direction is upstairs, and it is validated due to the nearest position from the trajectory starting point. In the vertical direction, a deviation error is recognized using vertical section profiles along the building sleeve of only $2 \mathrm{~cm}$ also in the central vaulted module; about $2 \mathrm{~cm}$ in the thickness of the floor of the overlying room, and up to $4 \mathrm{~cm}$ in the higher and farthest part or the large room above. This is generally due to the lack of a vertical closure of the scanning trajectory also in the northern part of the building, and in the SLAM algorithm processing the constraints deriving from the intersection of common vertical and planar paths are thus missing. For this reason, the room above behaves cantilevered without a constraint in $\mathrm{Z}$.

Table 1: The deviation error in the control points used as rigid roto-translation. The vector value is split into the axes component.

\begin{tabular}{c|c|c|c|c} 
& $X(m)$ & $Y(m)$ & $Z(m)$ & Dist $(m)$ \\
\hline mean & 0.012 & 0.020 & 0.012 & 0.024 \\
st.dev & 0.013 & 0.029 & 0.007 & 0.013
\end{tabular}

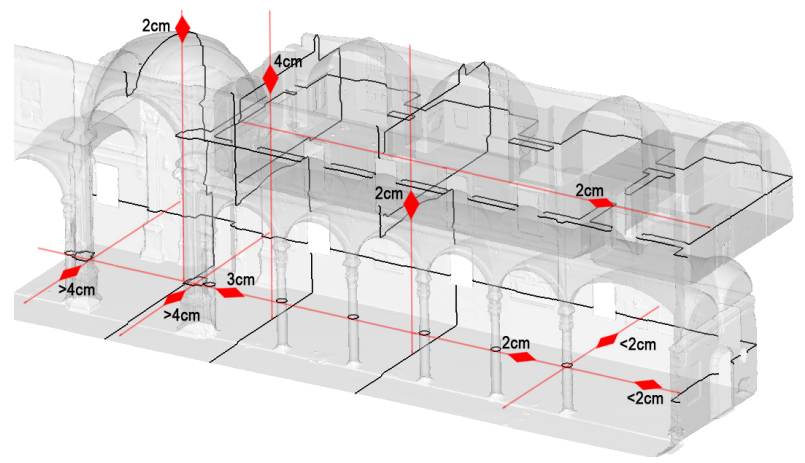

Figure 6: Drift error along the SLAM scan measured in deviation error between TLS and SLAM data.

The analysis related to geometric features of the point cloud data from a local point of view considers now the quality of objects reconstruction finalized to GOG9 and GOG10 and starts from the planarity, the density, the roughness, the curvature.

The perimetral wall of the Canonica portico is considered for planarity analysis to verify the ability of the two different point cloud, using region growing algorithm (Besl \& Jain, 1988; Vo, Truong-Hong, Laefer, \& Bertolotto, 2015) identifying points with similar characteristics of planarity starting from featuring seed points. This allows a comparison between SLAM data e TLS data to be intercepted by a fitting plan extracted and in Figure 7 is visible the result for the SLAM point cloud deviation map in scalar colours. The graph shows the distribution for both the dataset and displays that only $36 \%$ of points fits in $\pm 1 \mathrm{~cm}$ form the plan for SLAM, and $53 \%$ for the TLS data, but for both cases almost the $84 \%$ of points is within $\pm 3 \mathrm{~cm}$ from the plan. This implies, as expected for TLS sensors data and also for SLAM-based point cloud, an effortless recognition and extraction of plans for smoothed and generally regular areas as the single- 
curvature surfaces and walls, for their possibility to be parameterized in the model.
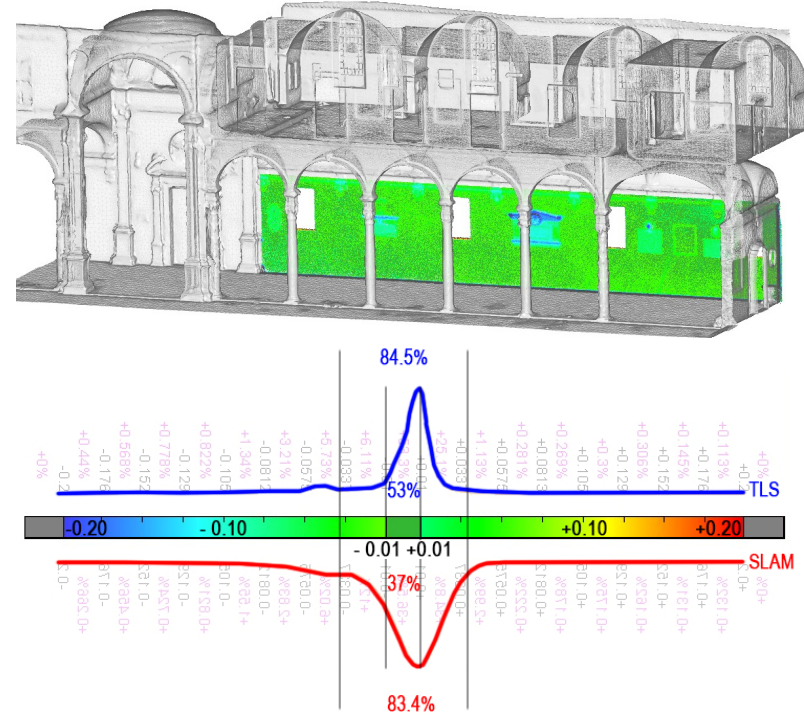

Figure 7: Plane fitting on points model from SLAM-based acquisition. In the related graph, the points distribution within \pm 1 $\mathrm{cm}$ for red SLAM, $37 \%<1 \mathrm{~cm}$; blue TLS, $53 \%<1 \mathrm{~cm}$.

\subsubsection{Density of data: between acquisition planning and post-processing optimization}

The concept of density of data is a crucial issue and is strictly related to the effectiveness of usage, both in the data acquisition planning and in data optimization for modelling procedures, whether these are based on triangulation modelling, or benefits from parametric geometry rules. Moreover, the density slightly changes implication and shows us different matters, if considered from the local and global point of view. In fact, the concept of density of information, from a global point of view concerns also the concept of continuity and completeness of data, as well as the quality of that.

In the case of SLAM-based 3D mapping, the distribution and overlapping of data are connected to typical operator walking point-of-view and the differences in density depending on the trajectory trend. In Figure $8 a$ the density gradient map of ZEB Revo system represented in the 3D environment along the trajectory, expressed with the number of neighbours in a pre-determined sphere (Algorithm implemented in CloudCompare [CC] software interface). It is the representation of the number of points contained in a local neighbourhood of a sphere with a $0.05 \mathrm{~m}$ radius (CloudCompare, 2015). Blue areas contain on average less than 100points in $0.05 \mathrm{~m}$ radius sphere (r.sph.), and denser areas are located at operator height. If the porch floor is digitized with almost 50 pts $/ 0.05 \mathrm{~m}$ r.sph., the topmost part of the façade in the connection with the moldings contains only 5 points. The point density in the $1^{\text {st }}$ floor rooms is almost $100-200$ pts $/ 0.05 \mathrm{~m}$ r.sph. and the values exceed the 300 pts only where the operator came very close to surfaces (red). The uniformity, as in Figure $8 \mathrm{~b}$, is however guaranteed, opposite to the static TLS, by the possibility for the operator to cover all the accessible spaces even with the extension of arms or supporting tools, and so this decreases considerably with the height.

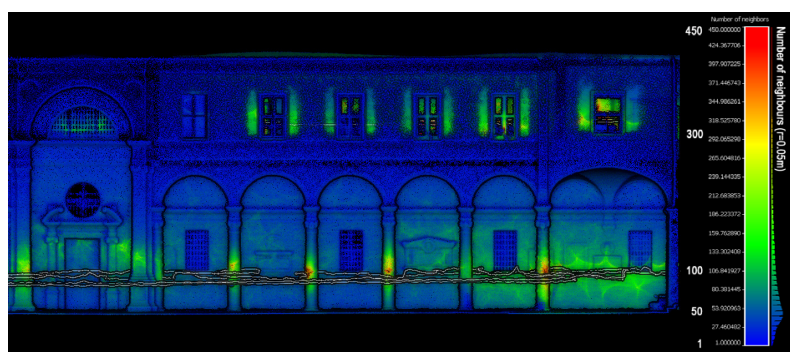

(a)

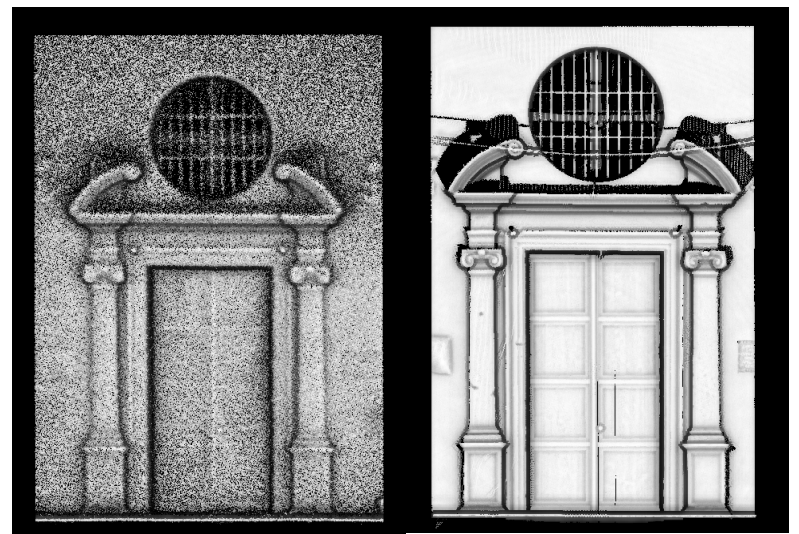

(b)

Figure 8: (a) Density distribution with ZEB Revo acquisition along the trajectory (white line), with typical operator-point-ofview. (b) Comparison between shadow areas in static TLS and continuity in SLAM dataset.

The major local density of TLS point clouds is featured by a general non-uniformity of points distribution depending on overlapping areas between scans. It frequently causes a lack of data if shadow areas occur in Figure 8b. They are above the frames or in the presence of interposed objects that cause shadow areas, which are only covered by the correct planning of other scan positions. Given the bottleneck of the LiDAR data weight for traditional TLS scans (up to $1 \mathrm{~Gb} / \mathrm{scan}$ with colour value), if on one hand, the increase of scan positions guarantees a greater coverage and density uniformity for the scanned surfaces, on the other hand, it increases exponentially the amount of data, negatively affecting the manageability of the scans project. In Figure 9 the final optimal distribution of the scans necessary in the ground floor for the indoor/outdoor surfaces, with a simulation carried out by using the methodology presented in (Díaz-Vilariño, Frías, Previtali, Scaioni, \& Balado, 2019) and develop in Par.5.

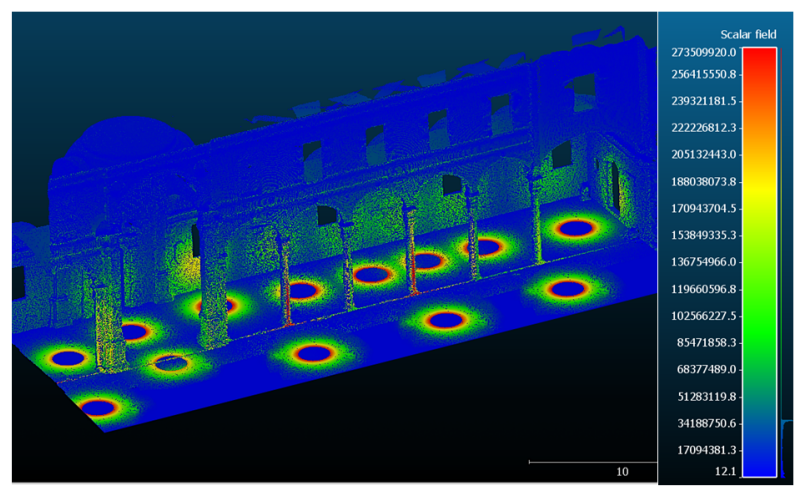

Figure 9: Optimized distribution of scans ensuring maximum uniform overlapping and minimum number of acquisitions. 


\subsubsection{Details and precision of point clouds}

In order to evaluate the Level of Detail that the digitization methodology can satisfy using the two scanning solutions, a specific analysis should be conducted, typically for architectural elements as the ones of the Canonica arcades of Bramante. The analysis is based on the 3D features that commonly define geometric description of point cloud surfaces, helping the possibility of their segmentation and classification (Grilli, Menna, \& Remondino, 2017; Paiva, Cogima, Dezen-Kempter, \& Carvalho, 2020). They are not only the values from density computation methods but also the curvature analysis of points surface, roughness of points distribution in order to evaluate the optimal semi- or automatic recognition of continuous surfaces, of edges and break lines.

The local density analysis (Fig. 10) focused on the upper part of the column shaft and the capital underlines the remarkable difference in detail definition for data (a) and (b) with a 3:1 ration for density mean value magnitude.
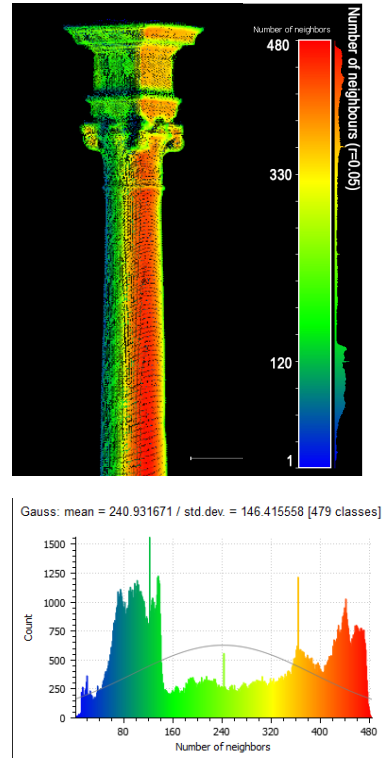

(a)
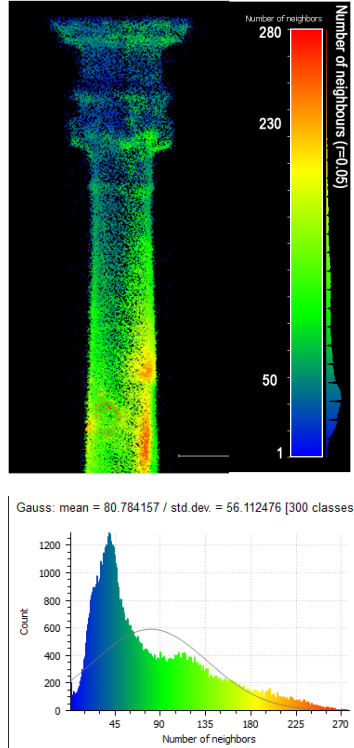

(b)
Figure 10: Analysis of details content in the column element Density comparison by number of neighbours [CC] $(r=0.05 \mathrm{~m})$ for TLS (a) and SLAM (b).

The curvature analysis is able to highlight, in the other side, the surface detail trend in a 'kernel' size, that is the radius of a sphere inside which the neighbours are extracted (around each point) and evaluated for the curvature. This computation is very sensitive to noise and outliers' values ([CC], 2015). In fact, as stressed in the assessment of Figure 11, if a $3 \mathrm{~cm}$ kernel radius is able to fit the centimetric detail of the capital, a kernel radius of $15 \mathrm{~cm}$ is rather necessary for emphasize the architectural order details for the abacus and pulvino.

Therefore, the two surfaces radically differ due to the noise errors typically affecting the SLAM point cloud generation. It could be specifically evaluated with roughness analysis that is able to establish, according to a local neighbourhood defined as a sphere, the accepted points and outliers' values according to a distance (CloudCompare, 2015).
In Figure 12, the statistical distribution of points according to roughness method applied with a radius equal to $5 \mathrm{~cm}$ kernel size, clearly points out the homogeneity of TLS Lidar points precision (a), related to violet point color (outlier values only localized in corners description) and, in (b) the wider distribution of the curve, related to a greater distribution on the surface of noise points (green and yellow). This implies, when calculating a triangulated mesh surface, and/or in case of manual or semi-automatic extraction of edges and representative polylines and breaklines, a greater effort in obtaining a smooth surface and simplified lines.

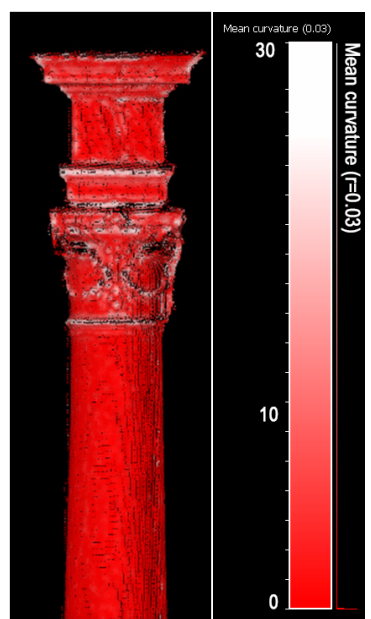

(a)

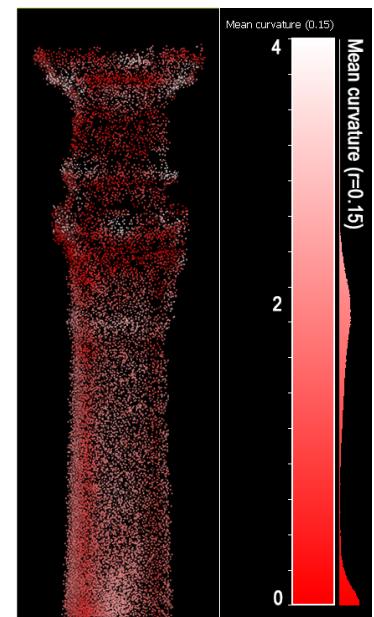

(b)
Figure 11: Curvature analysis highlighting detail elements with 0.03 kernel sphere for TLS data (a) and with 0.15 kernel sphere for SLAM point cloud (b)
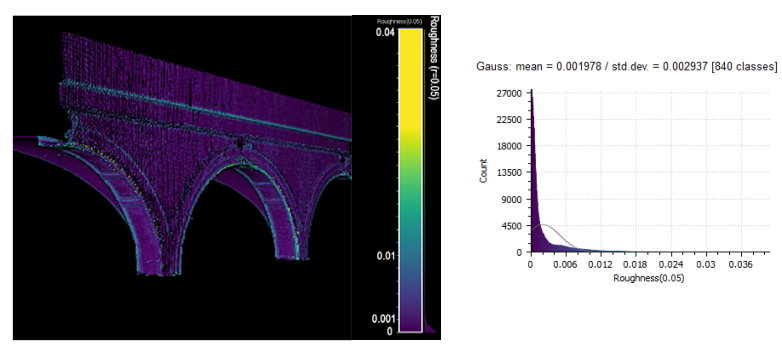

(a)
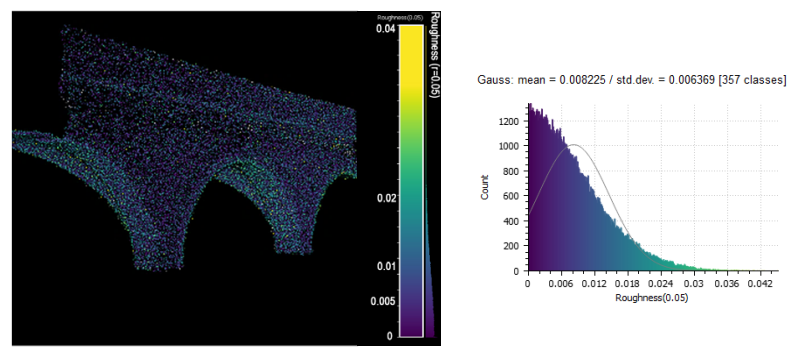

(b)

Figure 12: Noise error evaluation using roughness analysis with kernel sphere $(r=0.05 \mathrm{~m})$ for TLS $(a)$ and SLAM $(b)$.

\subsubsection{The vaulted surface representation}

Considering the single or double curvature surfaces, they generally require, according to the expect LOD, an interpolated surface that could be able to describe accurately not only the generative geometry but also even 
local irregularities or structural deformation from original shape. Beyond the pure triangulation modelling based on the optimized point cloud, a GOG10 is thus the proper generation solution based on points sample interpolation within an exterior closed edge. Sometimes probably the GOG9 is also admitted, starting from a set of polylines in the form of extracted profiles and even isolines to represent the local irregularity, within the closed edge.

The use of extracted profiles and sub-samples of dense points is almost established by recent application in researches based on the use of static LiDAR data. But what happens if a lower precision point cloud as an initial data takes over, as the SLAM-based here? In the research here presented, it was explained how the geometric features are radically different and also the designated modelling strategy could change. The SLAMbased point cloud, as shown in Figure 13 is featured by inherent variability in points distribution in comparison with TLS data. However, it could be stated that in the case of the circular vault sample in the central module of the porch, $90 \%$ of the points are comprised within $2 \mathrm{~cm}$ error, and only $57 \%$ has a deviation of almost $1 \mathrm{~cm}$.
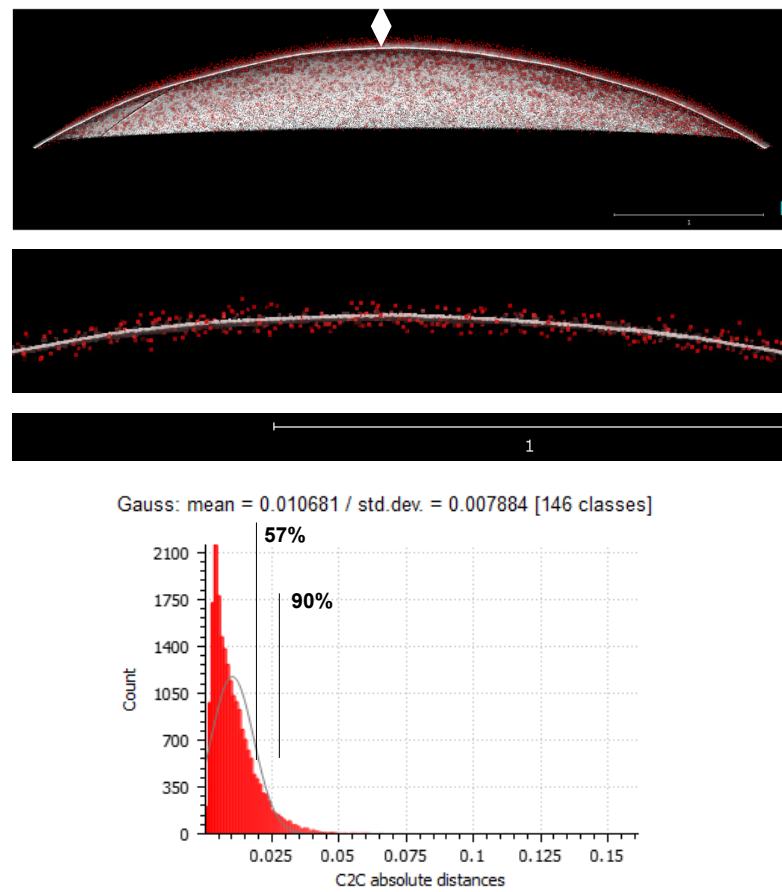

Figure 13: Surface deviation analysis Cloud to Cloud [C2C] between TLS surface and SLAM-based data, and the distribution of the distance on the related graph.

As expected, and as it will be demonstrated in the next paragraph, this noise badly influences the direct parametric generation in the case of NURBs interpolation by GOG 10 and so GOG 9 approach is parallelly presented. Structure isolines and eventually isolines are convenient in the GOG9 pipeline. In the case of SLAMbased data, the proposed procedure must go through the calculation of a triangulated surface.

For this reason, and generally, if a mesh model is required as the first step of modelling procedure for independent use, the triangulated surface of the vaulted elements is presented (already introduced in Figure 2, the groin lunette vault). From the calculation of mesh model, both for TLS data (3.736.984 points; 404.657 triangles; tot. 15 min time) and SLAM-based data (396.241 points; 55.342 triangles; tot. 10 min time) a set of internal section curves and a set of isolines has been extracted with $0.05 \mathrm{~m}$ steps. In Figure 14 the isolines only are represented together for TLS data (blue) and SLAM-based data (red). Considering this vaults reconstruction, the test of isolines comparison emphasized a lack of detail up to $7-8 \mathrm{~cm}$ in the diagonal rib detail. This could be admitted if the assumptions in the planning phase of the parametric modelling generation are related to such LOD and scale.

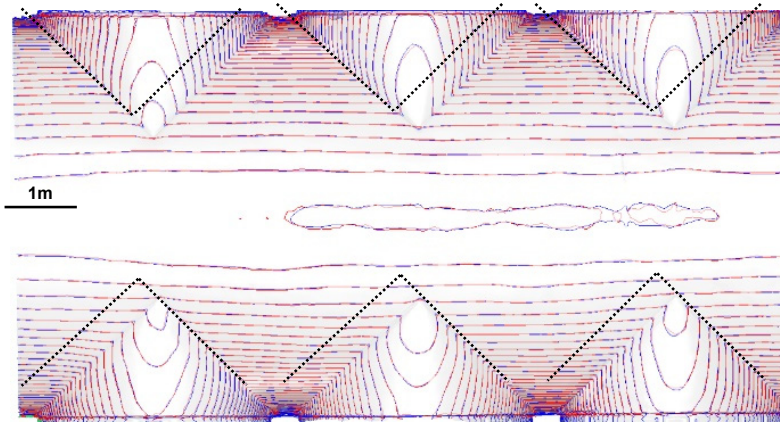

LiDAR data SLAM-based data

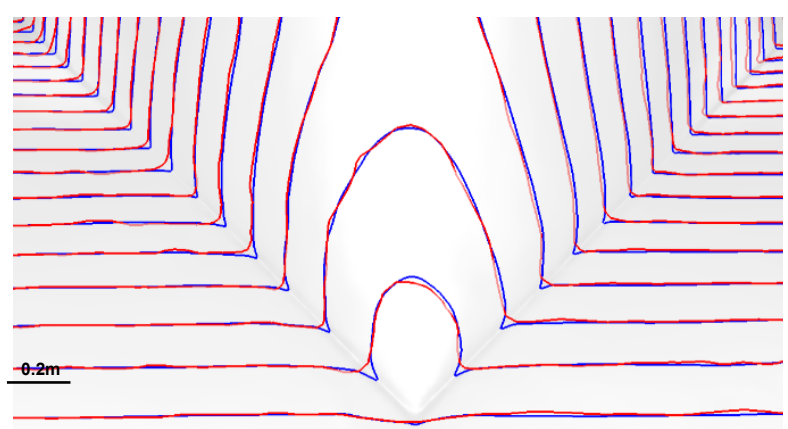

Figure 14: Extraction of isolines profiles ( $0.05 \mathrm{~m}$ step) and an indication of the diagonal ribs directions.

\subsection{Oriented parametric modelling generation}

Starting from the optimized point clouds originated from the two different scanning methods, and wherever necessary the 3D wireframe polylines extracted from the 3 samples considered for the analysis, the following workflow has been implemented. The samples are:

a) The circular vault in the central higher module of the arcades.

b) A column-type of the porch.

c) An arch-type connecting the columns of the porch.

From the cleaned and filtered point cloud, a set of primitives and edges have been extracted from the 3D point cloud (3D mesh model for SLAM data). The NURBS models have been calculated with GOG9 for A, B, C, and GOG10 for A. For the parametric NURBS models results the GOA was analysed and verified (Banfi, 2020) according to the starting point data.

\subsubsection{The circular vault}

For the parametric modelling of the circular vault with SLAM data, the GOG9 and GOG10 were implemented and evaluated. The NURBS models based on TLS data are the reference ground truth (around 700.000 points for 
the circular vault). The GOG10 was executed using two sub-sampled point clouds: a first-step filtering sample of points (35000 points) and an onerous sub-sampling of about $1 / 30$ points (Table 2 ). The GOG9 was tested with an external edge and isolines with $0.10 \mathrm{~m}$ and $0.20 \mathrm{~m}$ step. In order to verify data accuracy, the four parametric surfaces, are measured with their GOA and st. dev. compared with the original TLS points dataset. In Table 2 the results are expressed in terms of the percentage of points within $1 \mathrm{~cm}$ and $5 \mathrm{~mm}$. In the end, no substantial difference in the final surfaces emerged, and in any case not largely exceeding $1 \mathrm{~cm}$.

Table 2: The GOG parametric modeling applied to the central vault based on SLAM-data.

\begin{tabular}{|c|c|c|c|}
\hline \multicolumn{3}{|c|}{ GOG - Grade of Generation tests } & \multirow{2}{*}{$\begin{array}{c}\begin{array}{c}\text { GOA (points } \\
\text { deviation) }\end{array} \\
86,7 \% \pm 0.5 \mathrm{~cm}\end{array}$} \\
\hline GOG10 & 10.1 & 35.600 points & \\
\hline & & & $94,1 \% \pm 1 \mathrm{~cm}$ \\
\hline & 10.2 & 1.360 points & $49,4 \% \pm 0.5 \mathrm{~cm}$ \\
\hline & & & $79,3 \% \pm 1 \mathrm{~cm}$ \\
\hline \multirow[t]{4}{*}{ GOG9 } & 9.1 & $0.10 \mathrm{~m}$ step isolines & $87,4 \% \pm 0.5 \mathrm{~cm}$ \\
\hline & & & $97,7 \% \pm 1 \mathrm{~cm}$ \\
\hline & 9.2 & $0.20 \mathrm{~m}$ step isolines & $55,9 \% \pm 0.5 \mathrm{~cm}$ \\
\hline & & & $82 \% \pm 1 \mathrm{~cm}$ \\
\hline
\end{tabular}

A further analysis was conducted on the parametric surfaces generated with the presented GOG. The choice was to use the RANSAC algorithm (implemented in CC interface) allowing the recognition and extraction of an adaptive spheric shape, firstly based on the starting points clouds and then on the generated NURBS. The extracted sphere dimension was around radius equal to $4.8 \mathrm{~m}$. For TLS point cloud, the $92 \%$ points fits within $\pm 1 \mathrm{~cm}$ in the sphere extraction, instead for SLAM points dataset, they are the $52 \%$. Applying the same algorithm to the parametric NURB surface with GOG9 and GOG10, that is an already approximated interpolation (verified with GOA yet), the RANSAC is able to extract the sphere almost with the $80 \%$ of surface both for TLS and SLAM NURBS. In particular, Figure 15 shows the GOG10.2 with the few point sample superimposed on the sphere and on the points dataset (black dots).

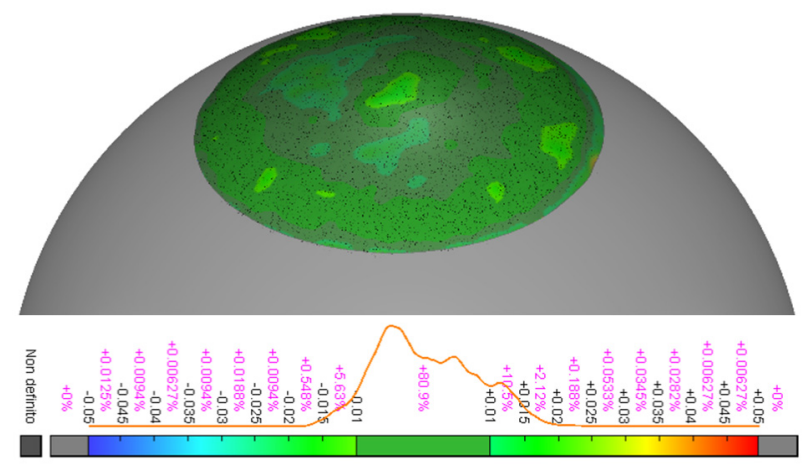

Figure 15: Deviation analysis between the reference sphere surface recognized with RANSAC. and the NURB derived from GOG10.2 applied to SLAM-data.

However, from the comparisons of the two GOG 10 it emerges that the lower precision of the SLAM data with the intrinsic irregularity, even with filtering the data, causes a non-smoothed surface for extended curved areas as vaults. The interpolation at step $20 \mathrm{~cm}$ can be added as the example in Figure 16 in comparison with the TLS surface where we consider the simplification in areas between curves, but we can define them however within the centimetres tolerance limits.
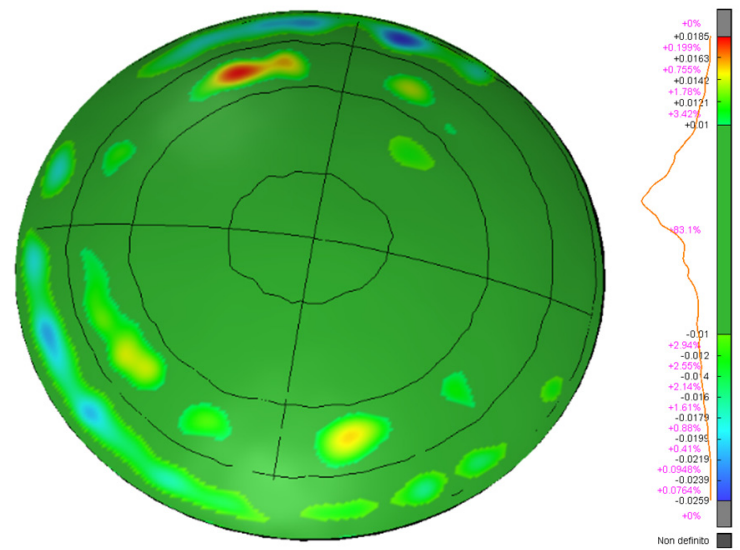

Figure 16: Deviation analysis between GOG10 applied to TLS (reference, higher detail expected) and GOG9.2 with $20 \mathrm{~cm}$ step profiles, applied to SLAM data (the poorest expected).

\subsubsection{The column}

In the case of GOG application for punctual elements as columns, it is well recognized the parametric approach based on GOG7-GOG9, or rather the binary swipe along the extracted profile. For the column generation based on the point cloud, the generatrixes and directrices extracted by parallel cutting planes applied to the 3D mesh model calculated on the SLAM data could be used: they can be employed as increasing constraints especially is required for the LOD, of if eventual anomalies of the geometry occurred. The test evidences the impossibility to use parametric as-built GOG to generate specific detail for basements and capitals as well. For them, focused modelling is required, and SLAM data does not support high level of detail for decorative apparatus as visible in next paragraph too (Fig. 17). Considering the column shaft and the entasis representation, for which the GOG9 is implemented here, the combined use of profiles as increasing constraints using the swipe method, as Figure 18 , could offer a solution according to the expected detail.

According to Figure 18, the (a) solution was employed in the generation of the parametric modelling from SLAM data, in comparison with NURBS generated with TLS reference data. In the GOA verification system, the GOG9 (a) results with $6.4 \%$ points with $\pm 1 \mathrm{~cm}$ deviation error from original point cloud data, and almost $90 \%$ with $\pm 2 \mathrm{~cm}$ deviation error, against $\pm 0.5 \mathrm{~cm}$ for TLS data. The major errors are positioned in the topmost and lowest area of the column entasis.

\subsubsection{The portal/arch}

In the case of the arch, the difficulty of the choice of GOG concerned the selection and construction of the 3D wireframe structure of the featuring polylines. The two parallel edge elements defining the arch between two columns are extracted semi-automatically from the mesh model calculated on SLAM data, necessary to recognize clearly edges due to noise affecting the surface (Fig. 12), 
and used to generate the parametric surface. After the GOA verification, the derived result from the comparison with the original point cloud declares that almost $57 \%$ of points are belonging to the new surface within $\pm 1 \mathrm{~cm}$, and in any case, almost $93 \%$ fits between $\pm 3 \mathrm{~cm}$. The use of an increased number of polylines constraints with the double swipe-based method along the external-internal edges, allows solving the problem of the lack of detail specifically located in the arch moulding modelling (Fig. 19).
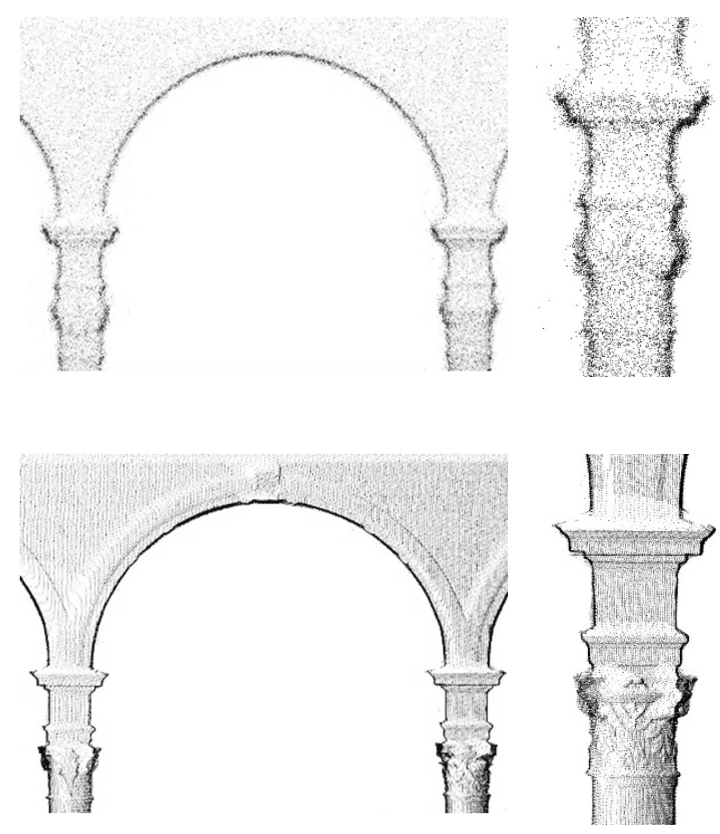

(a)
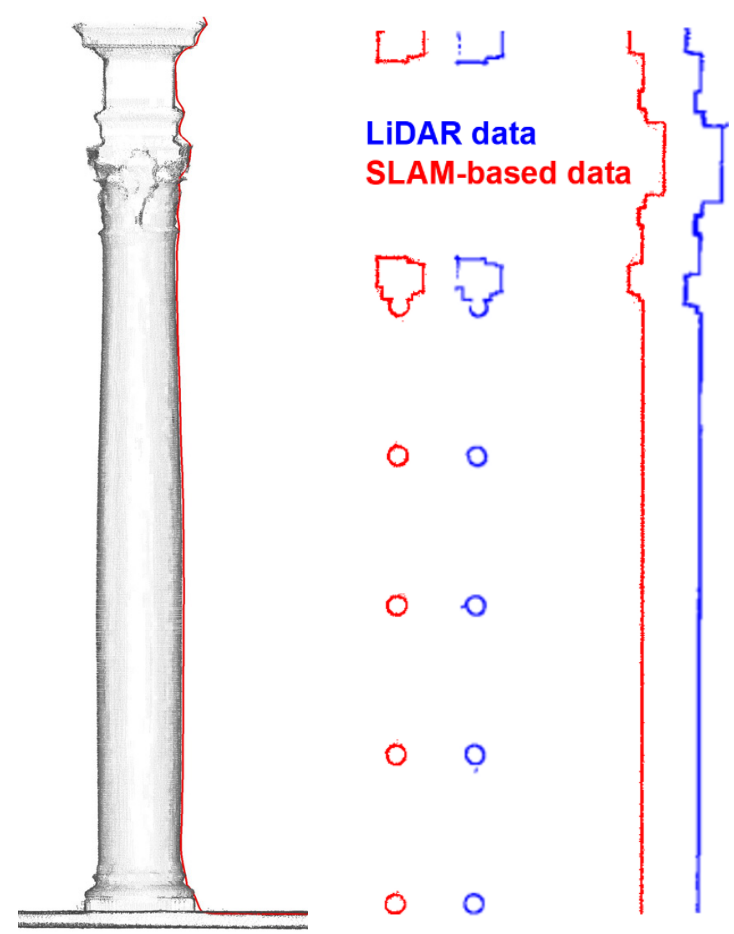

(b)

Figure 17: Data comparison between static TLS and SLAM data. (a) for façade digitization; (b) for profile extraction and digitization in vertical section and planar sections.

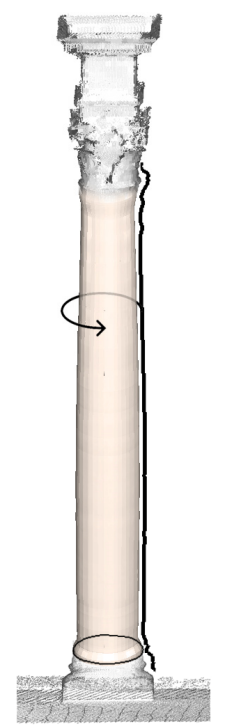

(a)

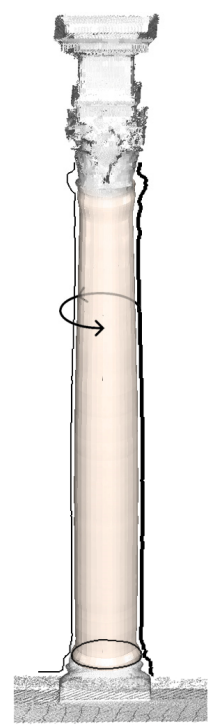

(b)

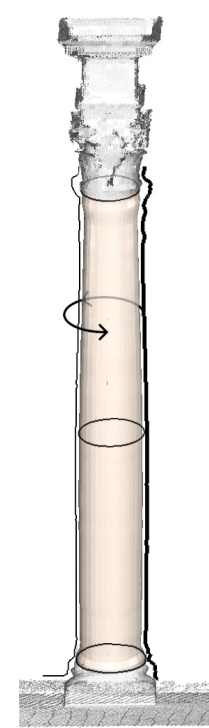

(c)
Figure 18: GOG9 swipe profile extracted from the column model. 1horiz/1vert. 1horiz/2vert. 3horiz/2vert.
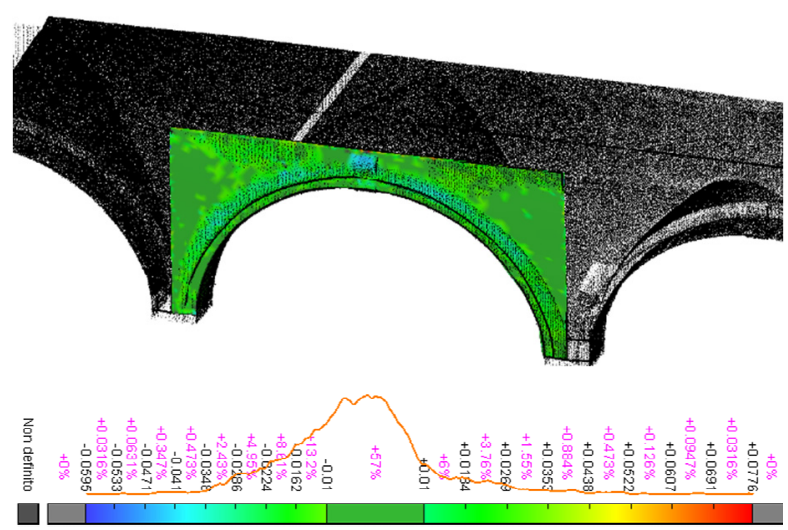

Figure 19: Deviation analysis GOA <between GOG9 applied to SLAM (higher detail expected) and original SLAM data

\section{Discussion}

The generation of parametric objects based on point cloud data it is part of a very well-established panorama as has been discussed in this work. The research demand, at the beginning of the validation analysis proposed and related to the test on the architectural elements of the S. Ambrogio Canonica court and arcades, is about the role of SLAM-based data in the HBIM modelling process. Due to the anticipated initial contradiction, the main goal is to establish how and when it is possible to place at the base of the consolidated scanto-HBIM pipeline a source data of a different nature, with lower density and precision, but with undoubted advantages in terms of efficiency of data acquisition and management times. The ZEB Revo point clouds are native with a lower level of detail $(<5-10 \mathrm{~cm})$ and for scales not greater than 1:100 (corresponding to the admitted error tolerance). But they can, in many cases, replace extensive and time-consuming use of static LiDAR for the generation of objects by BIM modelling or triangulated mesh models. This emerging portable digitization solution could be operationally suitable for 
many more survey purposes and applications than those for which the tool is commonly currently employed.

Table 3 tries to summarize the comparison analysis between the alternative existing approaches according to a set of variables introduced in the diagram.

Table 3: Preliminary comparison for the overall workflow for the two measurement systems

\begin{tabular}{c|c|c} 
& Static TLS & SLAM \\
\hline Data acquisition time & $* * *$ & $*$ \\
Data processing time & $* * *$ & $* *$ \\
Hardware-user work & $*$ effort & $*$ \\
Metric quality & $1: 20-1: 50$ & $1: 100-$ \\
& $1: 200$ \\
Precision & Edge and corners & Diffuse \\
noise & noise \\
Profile extraction & Point cloud & Mesh \\
Parametric generation & GOG9-10 & GOG9 \\
Triangulation mesh & $* * *$ & $* *$ \\
modelling & $* * *$ & $*$ \\
Data size & &
\end{tabular}

Specifically, for the individual nature of the point cloud data, as previously observed, it could be identified one of the main differences between LiDAR and MMS approach, which is the data acquisition planning and the quality of captured data. The two main variables are: efficient time to obtain a uniform and homogenous coverage in terms of point cloud densities. In fact, in order to have an estimation of the ideal number of TLS scans and consequently the optimum acquisition time necessary to achieve the same coverage of the acquisition with the MMS, a simulation was proposed in a second phase and here discussed. The simulation, as introduced in Par.4.1.1, was carried out by using the methodology presented in (Díaz-Vilariño, Frías, Previtali, Scaioni, \& Balado, 2019). This methodology is divided into three steps: (i) 2D optimisation of the scanning position according to a minimum point density threshold (ii) 3D simulation of the scan to verify the data completeness, (iii) re-computation of the scanning positions if the density threshold is not met somewhere. In this case, the threshold data density was the one derived from the MMS point cloud (average value of 10000pts/m2) and no predefined overlap is set among point clouds. Under these constraints, the final simulation foresees 12 scans for the porch and the outer façade and 8 scans for the first floor. The simulation was carried out considering as static TLS a Faro Focus X 130 laser scanner and as scanning time 1/5 (i.e., 28 million points). In terms of the requested time of acquisition, this turns in approximately 60 minutes of scanning time. Nevertheless, taking into account scanner movements for repositioning by operators, approximately 90 minutes can be estimated and without forgetting target placing and topographic measurements, 120 minutes for a reliable overall value. On the contrary, for the execution of the acquisition only by operator walking handling SLAM-based scanner, 20 minutes totally are consumed. Considering also possible failed SLAM scans, which not only occasionally happen in the initialization phase, an overall value of $30-45$ minutes can be spent for the SLAM-based mapping in such a large environment $\left(2500 \mathrm{~m}^{3}\right)$.

These considerations allow providing a framework of SLAM point clouds distinctive behaviour in meeting those requirements typical of the HBIM parametric modelling with Grades of Generation that we establish at the beginning as a pipeline well consolidated with TLS data. In particular, from one side, it has been demonstrated that the scan-to-HBIM pipeline and the modelling strategies (GOG) used for TLS can be generally adopted also for MMS data, with real advantages in terms of data acquisition and processing. In the other side, a distinction should be defined in the case of GOG9 and GOG10 applicability. The final performance of the test on GOG10 NURBS based on SLAM source datasets (after a firststep of cleaning and optimization), does not allow considering the SLAM point cloud, equally efficacious both for the GOG9 and GOG10 application. Since GOG10 has been created to support the generation of complex architectural elements from points model, the point cloud surface noisiness is a bottleneck for SLAM data to retrieve smoothed and accurate NURBS surfaces. In the time-cost evaluation, nonetheless, the time expense for the generation of the simplified mesh from SLAM data play a key role because, on the other hand, the profiles and edges for the GOG9 application are necessary to be extracted. As visible in Figure 17, in the case of 2D/3D digitization using SLAM-based point clouds, the operator is asked to apply a higher interpretative effort than the LiDAR data, and in any case, the different density and noise of the surface do not allow the achievement of the same scale and LOD. On the other hand, it is important to consider that the use of SLAM-based data proves to be easily manageable, as introduced, also in the generation of a first-step of modelling with a triangulated mesh (contrary to costly mesh generation on TLS, according to optimization time and the number of triangles). In Figure 20 an example of a triangulated model calculated after a point cloud optimization (20 min time expense, 1,41 mln triangles), of the whole volume of the Canonica related to the presented test. As a fist-step on modelling, the generation of a mesh from this kind of MMS dataset results more adaptable, easily manageable and texturizable with high-definition data (Sammartano, Spanò, \& Teppati Losè, 2019), in those cases where a continuous light model is required for virtual navigation, web application and data enrichment.

If we consider the generation of rapid and manageable points clouds as an efficient alternative to static methods, the use of SLAM-based data can be also proficiently employed in independent targets: ortho-projection and rasterization for $2 \mathrm{D}$ drawing and plotting of sections and façades Figure 17a, and also triangulated mesh modelling allowing to derive precise extracted profiles.

\section{Conclusion and future perspectives}

We are aware that more and more parameters come into play, from a user-oriented perspective, in the crucial moment in which the 3D survey is planned and specific techniques are adopted. This is usually established to gain expected results in the 3D reality-based model's generation. The premise of the research is based on the well-known bottlenecks referring to static TLS data and the required postprocessing phases leading to the filtering of a significant percentage of the acquired massive data. 
In fact, the long-time applications using consolidated TLS digitization, providing a plentiful source of data for HBIM modelling, with huge efforts of procedural time and waste of collected points, justify nowadays the research in the direction of a worthwhile alternative, using different kinds of clouds, as the MMS-derived, that already born lighter than about 1:10 and therefore do not necessitate to undergo processes of simplification and massive decimation. An evaluation of the required LOG and LOD is however always necessary in these cases where the use of portable scanners is competitive. The aim pursued in the point cloud processing and management, especially for this kind of SLAM datasets, is to be able to extract and focus the semantic content of objects and elements, supporting the segmentation and the modelling phase. The possibility to generate effortless BIM models through more fluid processes, must not make us forget the BIM space as a complex database of information. In this perspective, the research can investigate interesting application in the integration of direct radiometric information useful in HBIM models enrichment for knowledge and interpretation of architectural objects. This direct data augmentation could be based on the fusion of active/passive data simultaneously captured by integrated portable systems, or co-registered from different times, in a multi-temporal perspective aimed at information monitoring and updating.

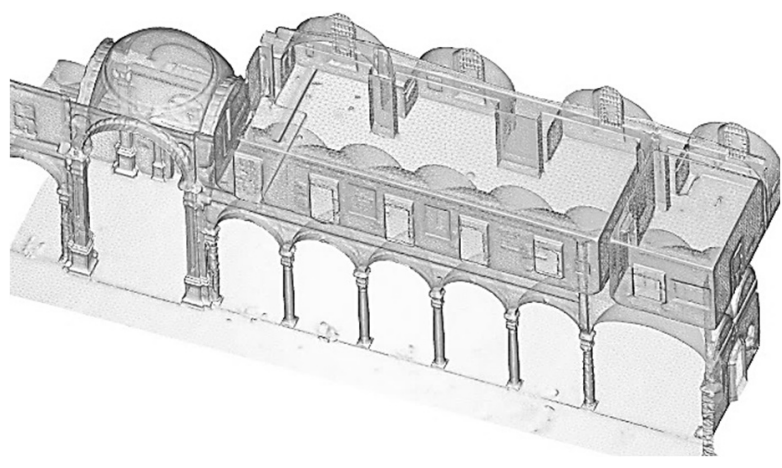

(a)

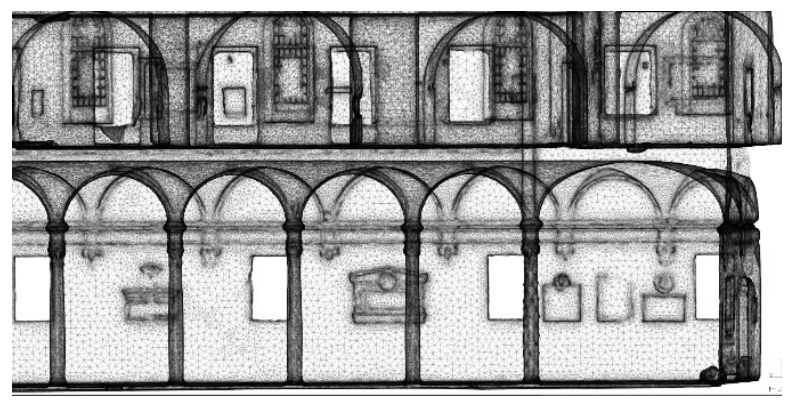

(b)

Figure 20: A low detail mesh model generated from the SLAM based data of the southern side or the Canonica porch $(70 \mathrm{Mb}$ ${ }^{*}$.obj data, without texture, for $2500 \mathrm{~m}^{3}$ of digitized space).

\section{References}

Banfi, F. (2017). BIM orientation: grades of generation and information for different type of analysis and management process. International Archives of the Photogrammetry, Remote Sensing and Spatial Information Sciences, XLII2/W5, 57-64. https://doi.org/10.5194/isprs-archives-XLII-2-W5-57-2017

Banfi, F. (2019). HBIM generation: extending geometric primitives and BIM modelling tools for heritage structures and complex vaulted systems. International Archives of the Photogrammetry, Remote Sensing and Spatial Information Sciences, XLII-2/W15, 139-148. https://doi.org/10.5194/isprs-archives-XLII-2-W15-139-2019

Banfi, F. (2020). HBIM, 3D drawing and virtual reality for archaeological sites and ancient ruins. Virtual Archaeology Review, 11(23), 16. https://doi.org/10.4995/var.2020.12416

Banfi, F., Brumana, R., \& Stanga, C. (2019a). A content-based immersive experience of Basilica of Sant'AMbrogio in Milan: from 3D survey to virtual reality. International Archives of the Photogrammetry, Remote Sensing and Spatial Information Sciences, XLII-2/W11, 159-166. https://doi.org/10.5194/isprs-archives-XLII-2-W11-159-2019

Banfi, F., Brumana, R., \& Stanga, C. (2019b). Extended reality and informative models for the architectural heritage: from scan-to-BIM process to virtual and augmented reality. Virtual Archaeology Review, 10(21), 14. https://doi.org/10.4995/var.2019.11923

Banfi, F., Previtali, M., Stanga, C., \& Brumana, R. (2019). A layered-web interface based on HBIM and 360 panoramas for historical, material and geometric analysis. International Archives of the Photogrammetry, Remote Sensing and Spatial Information Sciences, XLII-2/W9, 73-80. https://doi.org/10.5194/isprs-archives-XLII-2-W9-73-2019

Besl, P. J., \& Jain, R. C. (1988). Segmentation through variable-order surface fitting. IEEE Transactions on Pattern Analysis and Machine Intelligence, 10(2), 167-192. https://doi.org/10.1109/34.3881

Dewez, T. J. B., Yart, S., Thuon, Y., Pannet, P., \& Plat, E. (2017). Towards cavity-collapse hazard maps with Zeb-Revo handheld laser scanner point clouds. The Photogrammetric Record, 32(160), 354-376. https://doi.org/10.1111/phor.12223

Di Filippo, A., Sánchez-Aparicio, L., Barba, S., Martín-Jiménez, J., Mora, R., \& González Aguilera, D. (2018). Use of a Wearable Mobile Laser System in Seamless Indoor 3D Mapping of a Complex Historical Site. Remote Sensing, 10(12), 1897. https://doi.org/10.3390/rs10121897

Díaz-Vilariño, L., Frías, E., Previtali, M., Scaioni, M., \& Balado, J. (2019). Scan planning optimization for outdoor archaeological sites. International Archives of the Photogrammetry, Remote Sensing and Spatial Information 
Sciences, XLII-2/W11, 489-494. https://doi.org/10.5194/isprs-archives-XLII-2-W11-489-2019

Dore, C., \& Murphy, M. (2013). Semi-Automatic Modelling of Building Façades with Shape Grammars Using Historic Building Information Modelling. Proceedings of the International Archives of the Photogrammetry, Remote Sensing and Spatial Information Sciences - 3D Virtual Reconstruction and Visualization of Complex Architectures, XL(February), 57-64. https://doi.org/10.5194/isprsarchives-XL-5-W1-57-2013

Gatti Perer, M. L. (1995). La basilica di S. Ambrogio: il tempio ininterrotto (Vita e Pen).

Grilli, E., Menna, F., \& Remondino, F. (2017). A review of point clouds segmentation and classification algorithms. International Archives of the Photogrammetry, Remote Sensing and Spatial Information Sciences, XLII-2/W3, 339344. https://doi.org/10.5194/isprs-archives-XLII-2-W3-339-2017

Hichri, N., Stefani, C., De Luca, L., Veron, P., \& Hamon, G. (2013). From point cloud to BIM: a survey of existing approaches. International Archives of the Photogrammetry, Remote Sensing and Spatial Information Sciences, XL5/W2(SEPTEMBER), 343-348. https://doi.org/10.5194/isprsarchives-XL-5-W2-343-2013

Kumar, S. S., \& Cheng, J. C. P. (2015). A BIM-based automated site layout planning framework for congested construction sites. Automation in Construction, 59, 24-37. https://doi.org/10.1016/j.autcon.2015.07.008

Lusuardi Siena, S. (1997). Ambrogio, il costruttore sapiente. In M. Marco Rizzi, Cesare Pasini \& P. Rossignani (Eds.), La città e la sua memoria: Milano e la tradizione di Sant'Ambrogio (pp. 34-35). Electa.

Marucci, R. A., Negri, M., Rastelli, A. Romaniello, L. (2004). Bombe sulla città: Milano in guerra 1942-44 (Skira (ed.)).

Motawa, I., \& Almarshad, A. (2013). A knowledge-based BIM system for building maintenance. Automation in Construction, 29, 173-182. https://doi.org/10.1016/j.autcon.2012.09.008

Nocerino, E., Menna, F., Remondino, F., Toschi, I., \& Rodríguez-Gonzálvez, P. (2017). Investigation of indoor and outdoor performance of two portable mobile mapping systems. In F. Remondino \& M. R. Shortis (Eds.), Videometrics, Range Imaging, and Applications XIV, edited, SPIE 2017 (p. 103320I). https://doi.org/10.1117/12.2270761

Oreni, D., Brumana, R., Della Torre, S., Banfi, F., Barazzetti, L., \& Previtali, M. (2014). Survey turned into HBIM: the restoration and the work involved concerning the Basilica di Collemaggio after the earthquake (L'Aquila). ISPRS Annals of Photogrammetry, Remote Sensing and Spatial Information Sciences, II-5(June), 267-273. https://doi.org/10.5194/isprsannals-II-5-267-2014

Paiva, P. V. V., Cogima, C. K., Dezen-Kempter, E., \& Carvalho, M. A. G. (2020). Historical building point cloud segmentation combining hierarchical watershed transform and curvature analysis. Pattern Recognition Letters, 135, 114-121. https://doi.org/10.1016/j.patrec.2020.04.010

Remondino, F. (2003). From point cloud to surface: the modeling and visualization problem. International Archives of Photogrammetry, Remote Sensing and Spatial Information Sciences Remote Sensing, 34.

Rocha, G., Mateus, L., Fernández, J., \& Ferreira, V. (2020). A Scan-to-BIM Methodology Applied to Heritage Buildings. Heritage, 3(1), 47-67. https://doi.org/10.3390/heritage3010004

Sammartano, G. (2018). Optimization of Three-Dimensional (3D) Multi-Sensor Models For Damage Assessment in Emergency Context: Rapid Mapping Experiences in the 2016 Italian Earthquake. In F. Remondino, A. Georgopoulos, D. Gonzalez-Aguilera, \& P. Agrafiotis (Eds.), Latest Developments in Reality-Based 3D Surveying and Modelling (pp. 141-168). MDPI. https://doi.org/10.3390/books978-3-03842-685-1-7

Sammartano, G., \& Spanò, A. (2018). Point clouds by SLAM-based mobile mapping systems: accuracy and geometric content validation in multisensor survey and stand-alone acquisition. Applied Geomatics, 10(4), 317-339. https://doi.org/10.1007/s12518-018-0221-7

Sammartano, G., Spanò, A., \& Teppati Losè, L. (2019). A fusion-based workflow for turning slam point clouds and fisheye data into texture-enhanced 3D models. International Archives of the Photogrammetry, Remote Sensing and Spatial Information Sciences, XLII-2/W17(December), 295-302. https://doi.org/10.5194/isprs-archives-XLII-2-W17-2952019

Riisgaard, S., \& Blas M-R.. (2005). SLAM for Dummies. A Tutorial Approach to Simultaneous Localization and Mapping. MIT OCW.

Spanò, A. (2019). Rapid Mapping methods for archaeological sites. 2019 IMEKO TC4 International Conference on Metrology for Archaeology and Cultural Heritage, MetroArchaeo 2019, 25-30.

Stanga, C., Spinelli, C., Brumana, R., Oreni, D., Valente, R., \& Banfi, F. (2017). A N-D virtual notebook about the Basilica of S. Ambrogio in Milan: information modeling for the communication of historical phases subtraction process. International Archives of the Photogrammetry, Remote Sensing and Spatial Information Sciences, XLII-2/W5, 653660. https://doi.org/10.5194/isprs-archives-XLII-2-W5-653-2017

Tucci, G., Visintini, D., Bonora, V., \& Parisi, E. (2018). Examination of Indoor Mobile Mapping Systems in a Diversified Internal/External Test Field. Applied Sciences, 8(3), 401. https://doi.org/10.3390/app8030401

Vo, A.-V., Truong-Hong, L., Laefer, D. F., \& Bertolotto, M. (2015). Octree-based region growing for point cloud 
segmentation. ISPRS Journal of Photogrammetry and Remote Sensing, 104, 88-100. https://doi.org/10.1016/j.isprsjprs.2015.01.011

Wong, K., \& Fan, Q. (2013). Building information modelling (BIM) for sustainable building design. Facilities, 31(3/4), 138157. https://doi.org/10.1108/02632771311299412

Yang, X., Lu, Y.-C., Murtiyoso, A., Koehl, M., \& Grussenmeyer, P. (2019). HBIM Modeling from the Surface Mesh and Its Extended Capability of Knowledge Representation. ISPRS International Journal of Geo-Information, 8(7), 301. https://doi.org/10.3390/ijgi8070301

Zlot, R., \& Bosse, M. (2014). Efficient Large-scale Three-dimensional Mobile Mapping for Underground Mines. Journal of Field Robotics, 31(5), 758-779. https://doi.org/10.1002/rob.21504

Zlot, R., Bosse, M., Greenop, K., Jarzab, Z., Juckes, E., \& Roberts, J. (2014). Efficiently capturing large, complex cultural heritage sites with a handheld mobile 3D laser mapping system. Journal of Cultural Heritage, 15(6), 670-678. https://doi.org/10.1016/j.culher.2013.11.009 